Musées, Patrimoine et Culture scientifiques et techniques

$128 \mid 2010$

mars - avril 2010

\title{
Pour un musée national de la santé à Lyon
}

For a National Museum of Health in Lyon

\section{Wendy Atkinson}

URL : http://journals.openedition.org/ocim/174

DOI : 10.4000/ocim. 174

ISSN : 2108-646X

Éditeur

OCIM

Édition imprimée

Date de publication : 1 mars 2010

Pagination : 12-20

ISSN : 0994-1908

Référence électronique

Wendy Atkinson, «Pour un musée national de la santé à Lyon », La Lettre de I'OCIM [En ligne],

128 | 2010, mis en ligne le 01 mars 2012, consulté le 19 avril 2019. URL : http://

journals.openedition.org/ocim/174; DOI : 10.4000/ocim.174 


\section{Pour un musée national de la santé à Lyon}

Wendy Atkinson *

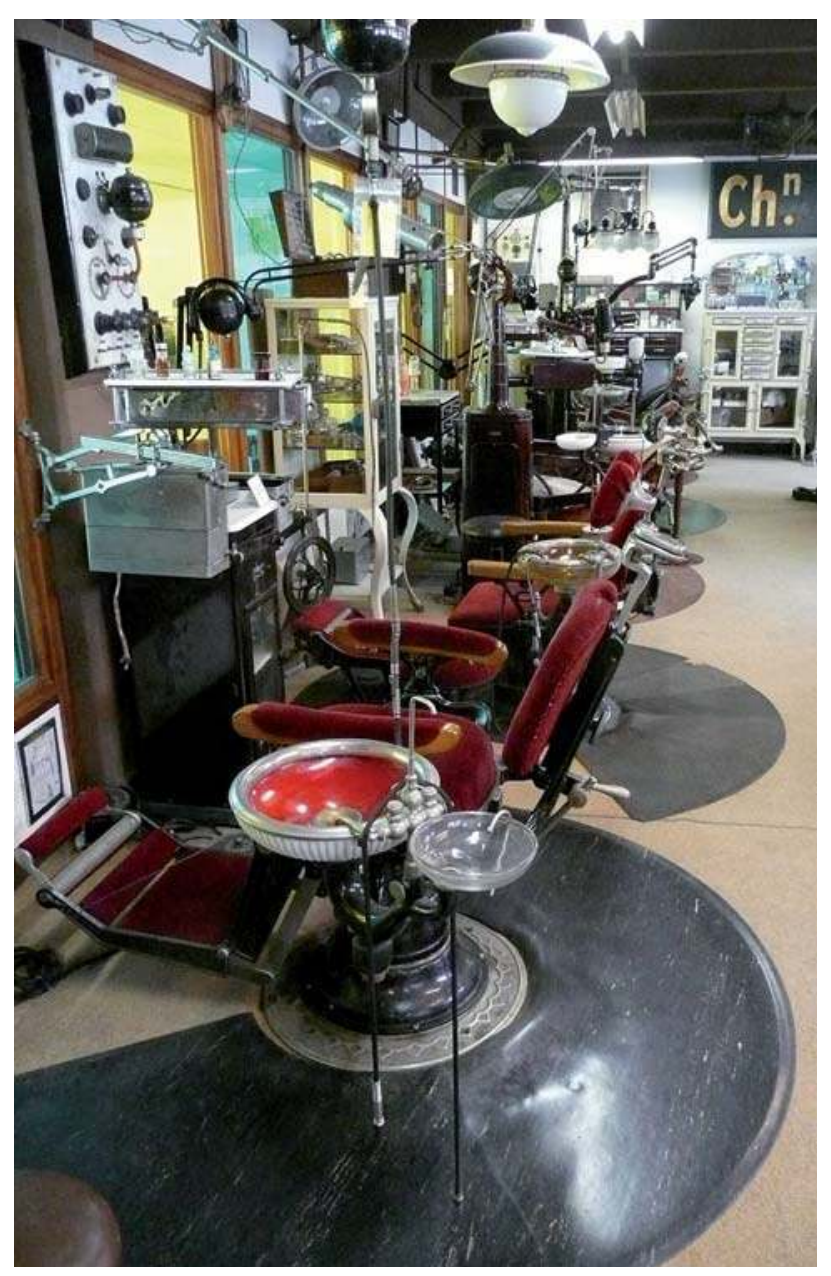

Les fauteuils dentaires alignés au sein du musée d'art dentaire de Lyon ๑) Musée d'art dentaire de Lyon/W. Atkinson

* Wendy Atkinson est étudiante en Master spécialisé Technologie, Culture, Patrimoine, Arts et Métiers ParisTech, Centre de Cluny wendyatkinson@free.fr
Un état des lieux des musées hospitaliers et de la médecine dressé à partir d'une enquête menée auprès d'établissements français et européens, et mettant notamment en lumière les atouts du contexte local, permet de pointer les différents éléments favorables à un regroupement des établissements dédiés à cette problématique en vue de la mise en place d'un musée national de la santé à Lyon.

L'Hôtel-Dieu de Lyon, propriété des Hospices Civils de Lyon depuis 1802, est en cours de désaffectation et les activités médicales s'arrêteront fin 2010 , ce qui suscite des interrogations sur le devenir de l'hôpital et de son musée actuel, ouvert au public depuis 1936. Un projet de convertir le musée des Hospices Civils de Lyon Hôtel-Dieu en un musée national de la santé, enrichi de collections industrielles et universitaires et ancré dans le site de l'Hôtel-Dieu (1), est en cours. Porté par le professeur René Mornex ${ }^{(2)}$, ce projet s'appuie sur un réseau de regroupement des musées lyonnais, universitaires et hospitaliers, et sur un conseil scientifique de préfiguration.

\section{La mise en place d'une enquête européenne}

Dans ce contexte, et dans le cadre d'une thèse professionnelle en technologie, culture et patrimoine, une mission d'étude sur le patrimoine de la santé à Lyon, en France, et en Europe m'a été confiée. Réalisée sous les auspices de Bruno Jacomy (3), cette enquête a été menée, commentée et exploitée 
entre avril et juin 2009. Elle devait remplir plusieurs objectifs :

- recueillir des faits et des chiffres sur quatre musées lyonnais partenaires du projet du musée ;

- présenter l'étendue du patrimoine de la santé à Lyon, dans le département du Rhône et la région Rhône-Alpes ;

- identifier le patrimoine français et européen en cherchant à décrire les institutions les plus représentatives ;
- réactualiser les connaissances (4) en faisant un état des lieux aussi exhaustif que possible sur le paysage européen du patrimoine de la santé ;

- justifier l'existence d'un musée national de la santé à Lyon, ville considérée comme l'épicentre de l'industrie pharmaceutique française, voire internationale, et le berceau d'innovations médicales notoires ; - proposer un début de réflexion sur le positionnement de ce même musée et faire ressortir les réactions au projet.

\section{Le potrimoine pris en comple dons l'enquête}

\section{Lyon et sa région proche}

- Musée des Hospices Civils de Lyon Hôtel-Dieu (MdF)

- La collection de radiologie Albert Renaud (association)

Trois musées universitaires et des fonds anciens de livres :

- Musée dentaire (faculté d'Odontologie)

- Musée de l'Histoire de la Médecine et de la Pharmacie (faculté de Médecine)

- Musée d'Anatomie et d'Histoire naturelle Testut Latarjet (association des sciences médicales de Lyon)

D'autres fonds anciens:

- ouvrages (Lacassagne, Boiron...) à la Bibliothèque municipale de Lyon

- ouvrages anciens de l'association des Internes à l'Hôtel-Dieu Des musées et des collections en périphérie:

- Musée de Sciences biologiques Docteur Mérieux (association) - Musée Lesbre-Tagand, École nationale Vétérinaire de Lyon (uniquement accessible aux étudiants de l'école vétérinaire)

- Musée Léopold Ollier (Ardèche) (municipal et association)

- Musée Claude Bernard (Rhône) (municipal et association) - collection criminalistique de l'École nationale supérieure de la Police à Saint-Cyr (fermée au public depuis 1982)

\section{France}

Paris :

- Musée Pasteur (Fondation Pasteur)

- Musée Fragonard de l'École vétérinaire de Maisons-Alfort (ministère de l'Agriculture et de la Pêche) (MdF)

- Musée de l'Histoire de la Médecine, Paris, université René Descartes (ministère de l'Enseignement supérieur et de la Recherche)

- Musée du service de Santé des armées au Val-de-Grâce (ministère de la Défense) (MdF)

- Musée de l'Assistance Publique-Hôpitaux de Paris (MdF) Le reste de la France:

- Musée Hospitalier, Charlieu (association et municipal) (MdF)

- Musée d'Anatomie (université Montpellier I)

- Musée de l'Hôtel-Dieu (Hospices Civils de Beaune)

- Musée grenoblois des Sciences médicales, Grenoble (association)

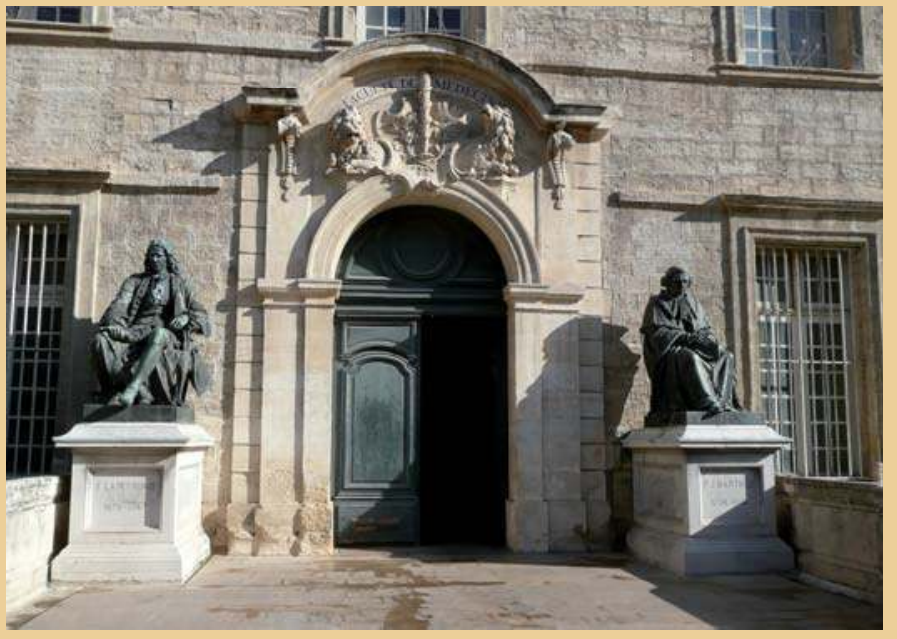

Le musée d'Anatomie se trouve dans l'un des bâtiments de l'université de Montpellier 1. (c) W. Atkinson

- Musée de l'École de Médecine navale, Rochefort (ministère de la Défense) (MdF)

- Musée Flaubert et d'Histoire de la Médecine, Rouen (CHU Rouen) (MdF)

- Muséum d'Anesthésie et des Techniques Médico-Chirurgicales, Besançon (association)

- Musée d'Anatomie et musée d'Histoire, Strasbourg (CHU et association)

\section{Europe}

- Allemagne : Berliner Medizinhistorisches, Museum der Charité (hôpital universitaire) et Dresde Deutsches Hygiene Museum (fondation)

- Angleterre : Science Museum, Londres (musée international, d'État) et Thackray Museum, Leeds (fondation)

- Belgique : Notre-Dame-à-la-Rose, Lessines (association)

- Danemark : Medicinsk Museion, Copenhague (universitaire) - Pays Bas : Boerhaave Museum (National Museum of the History of Science and Medicine), Leyde (musée national)

- Suisse : musée international de la Croix-Rouge et du Croissant-Rouge, Genève (musée international)

- Ukraine : National museum of Medecine of Ukraine (NMMU), Kiev (musée d'État, d'origine universitaire) 
Compte tenu des contraintes - l'importance du patrimoine en question, les délais de l'enquête, la disponibilité des conservateurs - il aurait été illusoire de faire un tour d'horizon complet. En dehors des musées lyonnais appartenant au réseau de regroupement, et me fiant aux recommandations des experts dans le domaine, Jacques Poisat (5) et Robert Bud (6), les musées français et européens ont été sélectionnés en fonction de leur représentativité : notoriété, diversité et exhaustivité de leurs collections, stratégies de diffusion et vocations scientifiques et culturelles.

Sur 41 musées contactés, 30 ont répondu : 9 européens, 13 français, 8 lyonnais, et 2 collections. Un questionnaire commun, en français ou en anglais, envoyé à chaque conservateur, portait sur : les activités, les collections (importance, type, grands secteurs de collection...), les surfaces (salles d'exposition, réserves, locaux administratifs), la fréquentation, les moyens humains et financiers, le profil et la provenance des publics, le type de gestion, l'utilisation de technologies, les programmes pédagogiques, l'approche muséale, les projets de rénovation, la communication et le marketing... Les données recueillies ont été complétées par des recherches supplémentaires, principalement sur Internet et à partir de visites sur place.

\section{Le patrimoine de la santé à Lyon}

Au Moyen Âge, Lyon est une ville de foires et de transit où le gîte, le couvert et les soins sont proposés. Son hôpital emblématique, l'Hôtel-Dieu, installé en centre ville depuis 1185, le long des quais du Rhône, accueille d'illustres personnalités : François Rabelais, Marc-Antoine Petit, Léopold Ollier, Antoine Gailleton, Alexandre Lacassagne, Étienne Destot, et, en l'absence d'université, assure la formation des médecins. En 1877, Lyon est doté d'une faculté de Médecine, tardivement par rapport à Montpellier, Toulouse et Strasbourg. Aujourd'hui, cette faculté fait partie de l'université Claude Bernard Lyon 1 et tisse de nombreux liens avec des hôpitaux, des laboratoires et des industries pharmaceutiques environnantes.

Commençant avec la cabinet de Marc-Antoine Petit, le patrimoine de la santé à Lyon et sa région s'articule autour de plusieurs axes : des collections pédagogiques et universitaires constituées au fil des années où pièces anatomiques et instruments médicaux jouaient un rôle essentiel dans la transmission des connaissances ; des souvenirs de la vie hospitalière, témoins du personnel médical et des pratiques de soins; des témoins de l'industrie et de la technologie

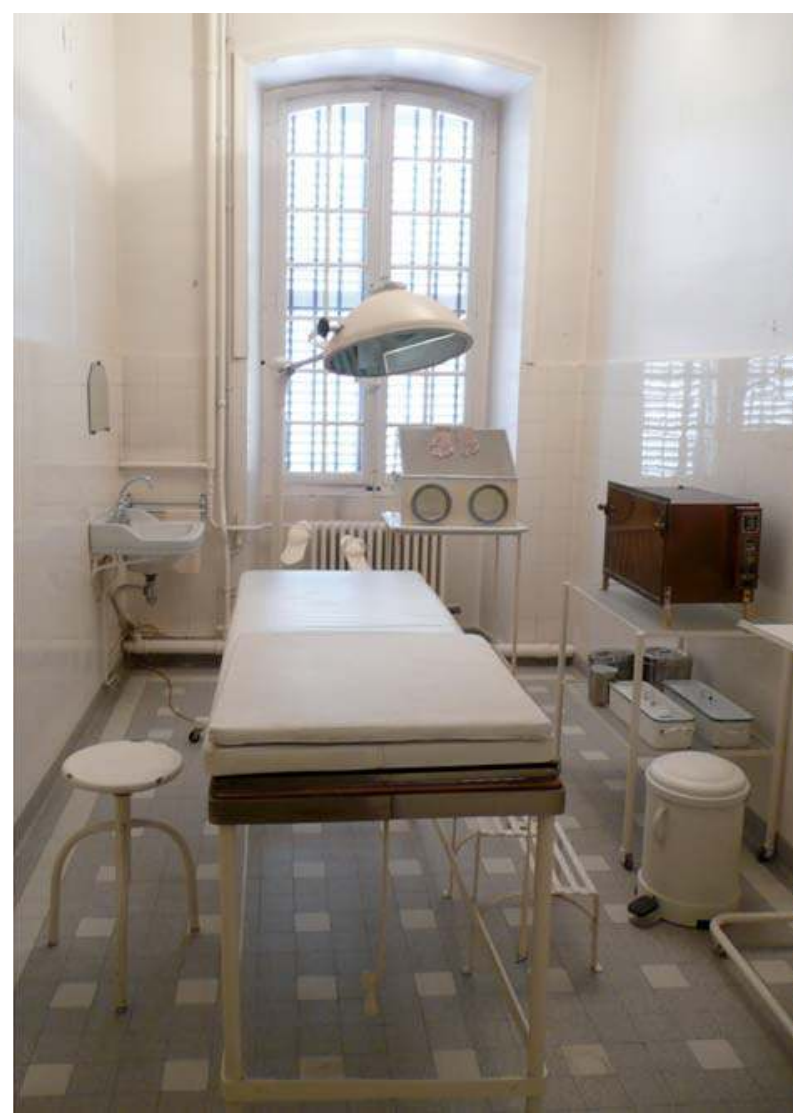

Une salle du musée hospitalier Charlieu (c) W. Atkinson

tel le musée des Sciences biologiques Docteur Mérieux ou la collection de radiologie Albert Renaud ; des apothicaireries ou maisons de médecins célèbres tel Léopold Ollier ou Claude Bernard; et diverses collections de documents et de livres anciens, propriété d'associations, de l'université ou encore de la municipalité.

Le projet de musée de la santé à Lyon ambitionne de rassembler sur un même site d'environ $9000 \mathrm{~m}^{2}$, au sein de l'Hôtel-Dieu, et à travers son exposition permanente des fonds anciens, des témoignages de la vie hospitalière, de l'évolution des soins et de la technologie, des pièces pertinentes des collections industrielles (radiologie) et pédagogiques (anatomie, dentaire et histoire de la médecine). Le programme scientifique et culturel, à travers des conférences et expositions temporaires, considérera les préoccupations contemporaines de la santé, les innovations et les personnalités marquantes de la médecine à Lyon et dans le monde. Lieu de ressources, point de rencontre entre sciences et société, ce musée réunira différents partenaires institutionnels et se veut un pivot de valorisation du patrimoine de la santé de la région Rhône-Alpes. 
Regardons de plus près les cinq piliers de la collection permanente de ce futur musée.

\section{Le musée des Hospices Civils de Lyon Hôtel-Dieu}

Fréquentation : 10000 visiteurs; labellisé « Musée de France »; propriété des Hospices Civils de Lyon ; surface d'exposition : $800 \mathrm{~m}^{2}$. Les 15000 objets, utilitaires ou de représentation, comprennent des tableaux, des vêtements liturgiques, des pièces d'orfèvrerie, des gravures et aquarelles, des vases à thériaque, 1200 faïences pharmaceutiques, 350 souvenirs d'enfants abandonnés, près de 2000 instruments de médecine et de chirurgie - stomatologie et moulage des « gueules cassées » - ainsi que 2500 livres anciens et des archives photographiques. Trois salles classées Monuments historiques de l'ancien hôpital de la Charité, détruit en 1934, ont été reconstituées de même que l'apothicairerie de l'Hôtel-Dieu.

\section{La collection de radiologie Albert Renand}

Accueilli par les Hospices Civils de Lyon depuis 2003, géré par l'association des Amis de la collection Albert Renaud, ce fonds de matériel de radiologie et d'électrologie contient 250 ensembles complets et près de 400 pièces uniques : appareils électriques, ampoules et tubes, films, plaques didactiques en verre, revues et livres anciens sur le début de l'électricité médicale des XIXe et XXe siècles. Les pièces remarquables comprennent un œuf électrique de Nollet, des roues de Ramsden et Winshurst, des tubes de Crookes et Coolidge...

Le musée d'Anatomie et d'Histoire naturelle de Lyon Testut Latarjet

Fréquentation : 10000 visiteurs par an, scolaires, étudiants et grand public ; propriété de la Société nationale de Médecine et des Sciences médicales de Lyon depuis 1921 (héritage en 1811 du cabinet de Marc-Antoine Petit) et accueilli par l'université Claude Bernard Lyon 1 au $3^{\mathrm{e}}$ étage du bâtiment Rockefeller ; surface d'exposition : $500 \mathrm{~m}^{2}$. Les 3000 objets, et 3000 ouvrages anciens, comprennent des éléments de la médecine légale et de la chirurgie expérimentale. Ce musée de l'anatomie pathologique et comparée offre un large panorama de préparations et présentations anatomiques, pièces conservées en bocaux, injections, corrosions, inclusions, anatomies naturalisées selon la technique de Fragonard, moulages démontables, en plâtre ou en carton, et cires anatomiques. Une collection importante de bassins rachitiques, des crânes phrénologiques, et des pièces de tératologie complètent le dispositif.

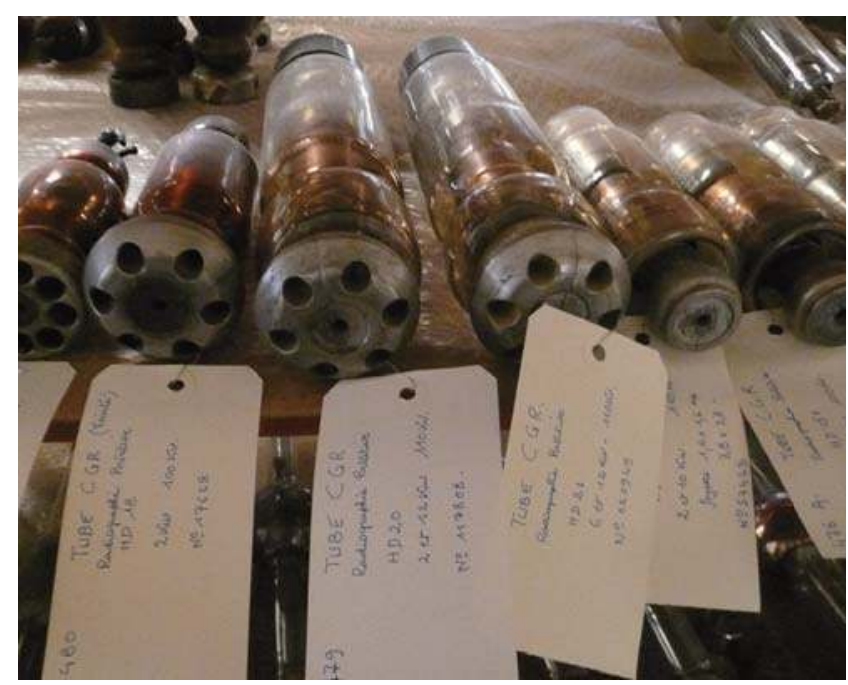

Une sélection de tubes de la collection de radiologie Albert Renaud (c) W. Atkinson

\section{L'histoire de la Médecine et de la Pharmacie}

Fréquentation : environ 1000 visiteurs par an, étudiants en médecine principalement ; surface d'exposition : $400 \mathrm{~m}^{2}$; propriété de l'université Claude Bernard Lyon 1, il est fondé par Alexandre Lacassagne en 1914. Situé au premier étage du bâtiment Rockefeller au sein de la faculté de Médecine Lyon-Est, ce musée expose des collections reflétant la transmission des connaissances médicales et l'évolution de la recherche à travers 3000 livres anciens et 5000 instruments et objets historiques. Tableaux et portraits des médecins titulaires des chaires de la faculté côtoient des crânes phrénologiques de Gall, un baquet de Mesmer, des appareils cinématographiques des Frères Lumière, la première seringue de Pravaz et de nombreux autres instruments, témoignages des travaux de Léopold Ollier, Claude Bernard ou Alexandre Lacassagne.

\section{Le musée dentaire}

Fréquentation : environ 700 visiteurs par an, étudiants et professionnels en Odontologie ; surface d'exposition : $200 \mathrm{~m}^{2}$; propriété de l'association Le musée dentaire de Lyon et hébergé par la faculté d'Odontologie de l'université Claude Bernard Lyon 1. Ouvert en 1979, ce musée, riche d'environ 3000 instruments et équipements - d'extraction, de prothèse, de fraisage ainsi que des fauteuils - complétés d'ouvrages anciens et de catalogues de fabricants, reflète l'évolution des techniques et de l'art dentaires entre 1860 et 1960.

Pour terminer ce tableau du patrimoine lyonnais, notons que seul le musée des Hospices Civils de 
Lyon Hôtel-Dieu (plein tarif : 4 euros) situé en centre ville, et ouvert du lundi au vendredi, de $13 \mathrm{~h}$ à $18 \mathrm{~h}$, bénéficie du label « Musée de France », dispose d'un budget (400 000 euros en 2008) et d'une équipe de huit salariés. Les trois musées universitaires, éloignés du centre, s'appuient sur des conservateurs bénévoles. D'accès restreint, seul le musée Testut Latarjet (tarif adulte : 2 euros) est ouvert de lundi au vendredi, tard le soir, et les samedis après-midi.

\section{Le patrimoine de la santé en France et en Europe}

Les deuxième et troisième volets de l'enquête sur le patrimoine de la santé en France et en Europe permettent de situer le projet d'un musée national de la santé à Lyon dans un contexte national, voire international. Cette mise en confrontation dégage les points forts des collections lyonnaises et souligne les avantages et les atouts du projet.

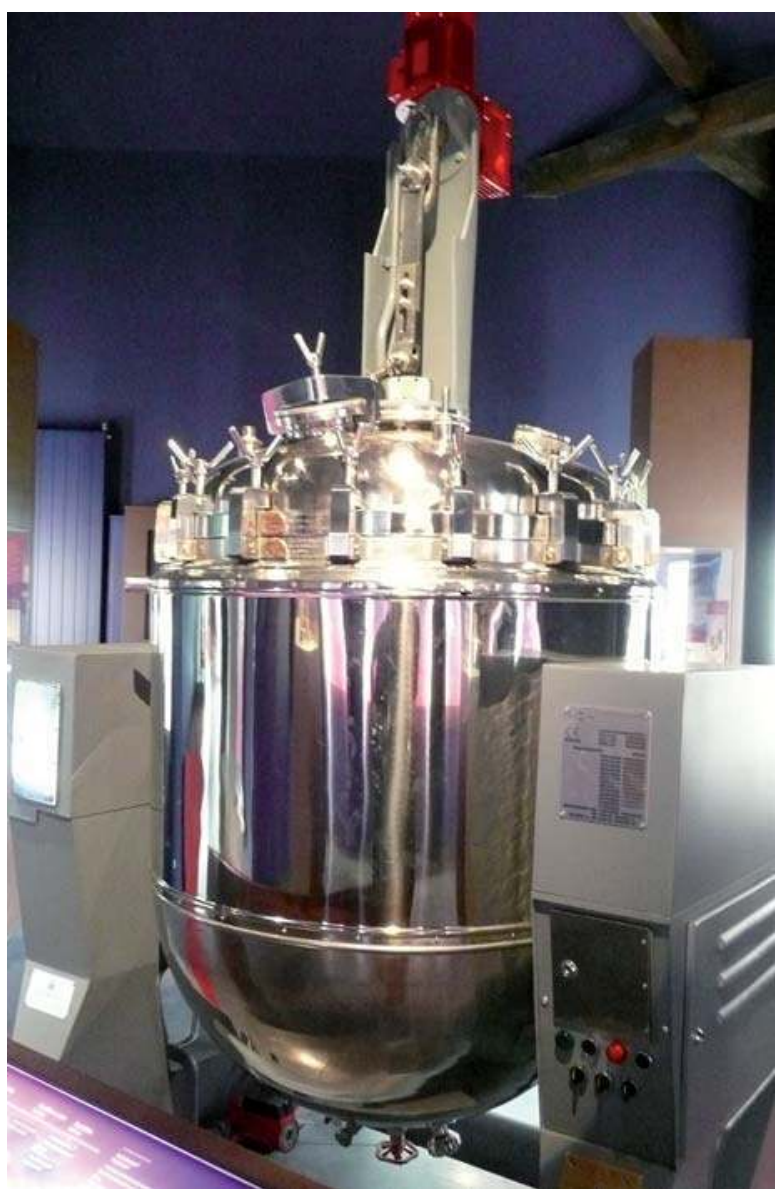

Un exemple du patrimoine du XXe siècle : un fermenteur de type Frenkel au musée de Sciences biologiques Charles Mérieux (c) W. Atkinson
En effet, même si plusieurs villes en France disposent d'un patrimoine de la santé - on dénombre au moins 75 institutions - il n'existe pas un musée national de référence en la matière. Quant à la situation en Europe, où l'analyse se limitait aux musées les plus représentatifs de sept pays, l'enquête a permis de mettre en lumière des pratiques muséales originales, leur fréquentation et fonctionnement, et de positionner le futur musée de la santé à Lyon dans leurs rangs.

Si les composants du patrimoine médical, hospitalier ou universitaire, en France, en Europe, se ressemblent - meubles, tapisseries, tableaux, salles d'apothicaireries, faïence pharmaceutique, objets hospitaliers, souvenirs d'enfants abandonnés, instruments de chirurgie, préparations anatomiques, cires et écorchés d'Honoré Fragonard ou Felice Fontana - chaque musée se distingue par une collection, un positionnement ou une stratégie de diffusion particuliers.

Parmi les établissements français, les musées universitaires participant à l'enquête semblent souffrir d'une pénurie de moyens. Le musée d'Anatomie de Montpellier ( $520 \mathrm{~m}^{2}, 6000$ pièces), fleuron du XIXe siècle avec ses planches anatomiques et ses exceptionnelles cires de Felice Fontana, restaurées en 2008, est fermé au public depuis dix ans. Le musée de l'Histoire de la Médecine (380 m², 3000 pièces), au sein de l'université Descartes à Paris, et qui relève du ministère de l'Enseignement supérieur et de la Recherche, raconte l'histoire de la Médecine à travers une esthétique du XVIIIe siècle et des objets de soins de Napoléon notamment.

La plupart des musées hospitaliers analysés bénéficient d'excellents emplacements au cœur de leurs villes : Charlieu (800 m², 1000 pièces), Rouen $\left(410 \mathrm{~m}^{2}\right)$, Beaune $\left(2325 \mathrm{~m}^{2}, 6000\right.$ pièces $)$, Paris $\left(1700 \mathrm{~m}^{2}\right)$, Grenoble $\left(450 \mathrm{~m}^{2}, 3000\right.$ pièces $)$, Besançon $\left(280 \mathrm{~m}^{2}, 300\right.$ pièces $)$. S'ils racontent tous la vie hospitalière, chacun a sa spécificité. Le musée des Hospices de Beaune, au cour du vignoble et sur un axe de circulation nord-sud, est le monument historique le plus visité de la Bourgogne ; avec sa grande chambre des malades du XVe siècle et ses tuiles bourguignonnes, il frôle les 400000 visiteurs par an. Le musée Flaubert et d'Histoire de la Médecine de Rouen, maison natale de Gustave Flaubert, dispose d'une importante collection d'objets des enfants abandonnés et l'étonnant mannequin d'accouchement de Madamme du Coudray. Quant au musée de l'Assistance Publique-Hôpitaux de Paris, la politique soutenue d'expositions temporaires permet d'exploiter les dispositifs muséographiques contemporains. 


\section{Principoles villes fronçaises possédant un musée de la Médecine}

En 2009, la France possédait plus de 75 musées de santé, hospitaliers, universitaires, communaux, privés, et des apothicaireries. Deux régions françaises portent une attention particulière à leur patrimoine de santé : RhôneAlpes et Bourgogne où de petits musées, hôtels-dieu et apothicaireries, ponctuent le paysage. Si, dans le passé, d'importants centres hospitaliers universitaires (Toulouse, Montpellier, Strasbourg, Nancy...) géraient leurs collections d'instruments médicaux et d'Anatomie, ces mêmes institutions peinent aujourd'hui à assumer cette mission.

\section{Nom du musée}

Hôtel-Dieu

Médiathèque, Maison Louis Pasteur

Hôtel-Dieu de Tournus

Hôtel-Dieu de Tonnerre

Musée de l’Hospice Saint-Roch

Conservatoire du patrimoine médical

Musée d'Histoire de la Médecine

Musée Curie, Musée Dupuytren, Musée Déjerine,

Musée des moulages de l'hôpital Saint-Louis

Musée du Centre hospitalier Sainte-Anne, Musée Cernea

Musée d'Histoire du Médicament, de la Pharmacie et de la Santé

Musée d'Anatomie

Musée de la Médecine

Musée de l'Hôpital d’Yssingeaux

Musée de l'Hôpital Saint-Jacques

Musée grenoblois des Sciences médicales

Pharmacie, Musée de l'Hôtel-Dieu-le-Comte

Apothicairerie d'Arbois

Hôtel-Dieu de Baugé

Hôtel-Dieu de Belleville

Apothicairerie de Bourg-en-Bresse

Apothicairerie de l'Hôpital de Chagny

Centre Hospitalier William Morey

Apothicairerie de Châtillon-sur-Chalaronne

Hôtel-Dieu et Apothicairerie de Lons-le-Saunier

Hôtel-Dieu de Louhans

Apothicairerie de l'Hôtel-Dieu de Mâcon

Apothicairerie de l'Ancien Hospice de Montluel

Apothicairerie de Saint-Amour

Apothicairerie de l'Hôpital de Salins-les-Bains

Hôtel-Dieu de Seurre

Apothicairerie de Thoissey

Hôpital Montpensier de Trévoux

Hôpital Saint-André

Trésors du musée de l'Hôtel-Dieu

Centre Hospitalier de Gap

Chapelle Sainte-Croix-de-Jérusalem, Hôpital Général de Dijon

Le musée Saint-Rémi

Chapelle Saint-Nicolas-de-l'Hôtel-Dieu

Musée de l'Hospice Comtesse

Musée régional hospitalier de Lille

\section{Ville}

Cluny (Saône-et-Loire)

Dole (Jura), ville natale de Louis Pasteur

Tournus (Saône-et-Loire)

Tonnerre (Yonne)

Issoudun (Indre)

Marseille (Bouches-du-Rhône)

Nancy (Meurthe-et-Moselle)

Paris

Châtenay-Malabry (Hauts-de-Seine)

Le Neubourg (Eure)

Hautefort (Dordogne)

Yssingeaux (Haute-Loire)

Les Andelys (Eure)

Grenoble (Isère)

Troyes (Aube)

Arbois (Jura)

Baugé (Maine-et-Loire)

Belleville (Rhône)

Bourg-en-Bresse (Ain)

Chagny (Saône-et-Loire)

Chalon-sur-Saône (Saône-et-Loire)

Châtillon-sur-Chalaronne (Ain)

Lons-le-Saunier (Jura)

Louhans (Saône-et-Loire)

Mâcon (Saône-et-Loire)

Montluel (Ain)

Saint-Amour (Jura)

Salins-les-Bains (Jura)

Seurre (Côte-d'Or)

Thoissey (Ain)

Trévoux (Ain)

Bordeaux (Gironde)

Château-Thierry (Aisne)

Gap (Hautes-Alpes)

Dijon (Côte-d'Or)

Reims (Marne)

Montreuil-sur-Mer (Pas-de-Calais)

Lille (Nord) 
Les deux musées de santé de l'Armée font cas à part. Situé dans l'ancien hôpital de la Marine, premier essai français d'architecture pavillonnaire et hygiéniste, le musée de l'ancienne École de Médecine navale à Rochefort (1 $000 \mathrm{~m}^{2}, 20000$ pièces) présente une importante collection de restes humains. Le musée du Service de santé des armées du Val-deGrâce à Paris $\left(1850 \mathrm{~m}^{2}, 850\right.$ pièces exposées, 13000 en réserves), au sein d'une abbaye du XVIIe siècle, inclut la magnifique chapelle dans sa visite. Outre les faïences, ce musée expose l'évolution des conditions sanitaires et de soutien aux armées au fil du temps.

Le musée Fragonard $\left(630 \mathrm{~m}^{2}, 5000\right.$ pièces, rouvert depuis 2008) dépend du ministère de l'Agriculture et de la Pêche. Il se situe au sein de l'École vétérinaire de Maisons-Alfort et expose les célèbres cires et écorchés animaliers de son premier professeur d'Anatomie, Honoré Fragonard.

En Europe, le musée Notre-Dame-à-la-Rose $\left(2800 \mathrm{~m}^{2}\right)$ de Lessines en Belgique, valorise son patrimoine bâti, cloîtres, réfectoire et dortoirs, et témoigne de la vie hospitalière à travers l'ancienne communauté religieuse.

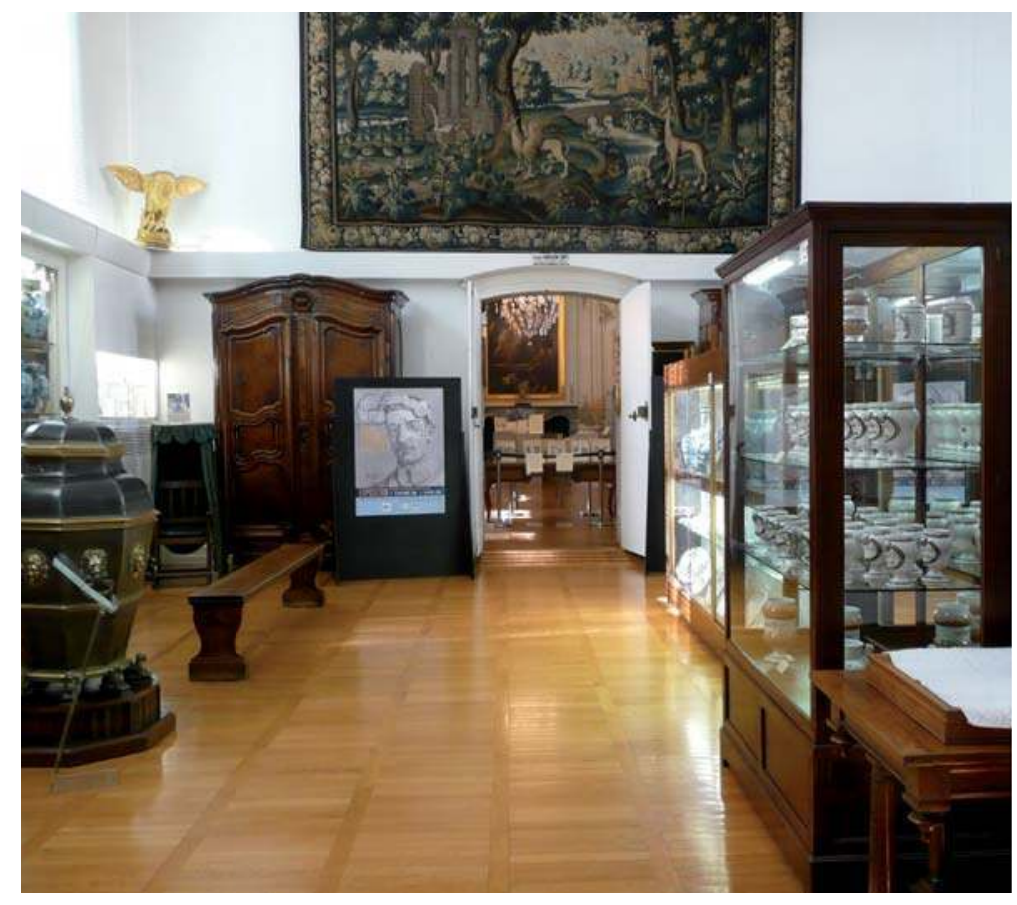

Une des salles du musée des Hospices Civils de Lyon Hôtel-Dieu () W. Atkinson
Le musée national de Médecine d'Ukraine ( $1400 \mathrm{~m}^{2}, 1500$ pièces exposées, 18000 en réserves), logé dans un monument historique au cœur de Kiev, raconte l'histoire médicale de l'Ukraine à travers une mixité de collections : beaux-arts, pièces d'anatomie... avec une volonté de raconter les désastres sanitaires du XXe siècle, tel l'accident de Tchernobyl, et d'accroître sa fréquentation par des activités artistiques.

Le musée de la Croix-Rouge et du Croissant-Rouge (3 $206 \mathrm{~m}^{2}, 240$ pièces exposés, 20000 en réserves), à Genève, musée international, se veut un lieu de rassemblement et de mémoire des conflits mondiaux. Ses archives des guerres sont inscrites au Registre de la mémoire du monde d'UNESCO.

Au Danemark, le Medicinsk Museion $\left(2150 \mathrm{~m}^{2}\right.$, 200 pièces exposées, 150000 en réserves), musée universitaire situé au centre de Copenhague, s'intéresse aux biosciences et intègre les technologies de communication communautaires (blogs, Facebook). L'Hygiene Museum (4 $500 \mathrm{~m}^{2}, 45000$ pièces) à Dresde, propose une histoire de la santé publique et des soins en Allemagne au $\mathrm{XX}^{\mathrm{e}}$ siècle à travers une politique d'expositions temporaires de renommée internationale.

Au Royaume Uni, les deux musées analysés étonnent à plusieurs égards. D'un côté le musée Thackray $\left(15300 \mathrm{~m}^{2}, 60000\right.$ objets exposés, soit $10 \%$ de la collection), legs de l'industriel Paul Thackray, raconte l'histoire de la Médecine d'un point du vue industriel et commercial. De l'autre, le Science Museum $\left(5000 \mathrm{~m}^{2}\right.$, plus de 100000 pièces en réserves), de vocation internationale, est dépositaire de plus de 30000 pièces provenant de la collection privée d'Henri Wellcome, contemporain et ami de Louis Pasteur.

Parmi les musées européens, quatre ont communiqué leurs budgets. Le musée Notre-Dame-à-la-Rose (10 salariés) gère un budget d'environ 600000 euros avec une part d'autofinancement entre 50 et $60 \%$; le musée Boerhaave (50 personnes), bénéficie d'un budget de 4 millions d'euros du gouvernement néerlandais ; le musée de la Croix-Rouge ( 15 employés) avec un autofinancement de $23 \%$, gère un budget constitué de dons et de subventions de 3 millions de francs suisses (environ 5 millions d'euros); le Thackray (26 employés) dispose d'un budget annuel autofinancé à $100 \%$ s'élevant à 750000 livres sterling (environ 830000 euros).

À Paris, les budgets sont moindres : 37000 euros, dont $80 \%$ en autofinancement, pour le musée 
Fragonard, un salarié et des élèves de l'École vétérinaire ; 300000 euros pour le musée du Val-de-Grâce, neuf salariés et une société externe pour l'accueil et la sécurité ; le budget du musée de l'Assistance Publique-Hôpitaux de Paris (14 employés) est fonction de son programme d'expositions temporaires et varie entre 110 et 400000 euros.

\section{Visiteurs et conditions de visites}

Tous les musées analysés attirent des publics similaires, en groupe ou individuellement : adultes, enfants, scolaires, étudiants, touristes, amateurs de l'histoire de la Médecine. Quelques musées attirent professionnels, congressistes, représentants de sociétés médicales et pharmaceutiques.

Parmi les musées européens, seul le Science Museum, subventionné à $90 \%$ par le gouvernement anglais, propose une entrée gratuite, sept jours sur sept. D’une fréquentation de 2,5 millions de visiteurs par an, la part dévolue à la Médecine, qui s'étend sur trois étages du bâtiment, est difficile à évaluer. Pour les autres, à l'exception du musée de Kiev (2,5 euros, 10-12 000 visiteurs), la tarification varie de 5 euros pour le moins cher (Museum der Charité, 50000 visiteurs) à 7,50 euros pour le plus cher (NotreDame-à-la-Rose, 40000 visiteurs). L'Hygiene Museum, qui pratique une tarification à la carte, 6 euros l'entrée unique, 16 euros l'année, accueille 280000 visiteurs par an tandis que les musées Boerhaave et Croix-Rouge, à tarification identique pour l'entrée adulte (6 euros), attirent 40000 et 100000 visiteurs respectivement.

Les musées français, à l'exception du musée de Beaune avec ses 380000 visiteurs en 2008 (plein tarif 6,50 euros), dépassent rarement les 10000 visiteurs/an. De la gratuité pour le musée des Sciences médicales de Grenoble (1 à 2000 visiteurs), la grille tarifaire varie de 3 euros pour le moins cher (Rouen, 11700 visiteurs) à 7 euros pour les musées Pasteur (9 à 10000 visiteurs) et Fragonard (10 000). La fréquentation du musée de l'Assistance publiqueHôpitaux de Paris oscille entre 16000 et 23000 visiteurs selon les expositions temporaires.

\section{Stratégies et politiques de diffusion}

Le Science Museum poursuit une politique d'exposition d'objets contemporains. Son installation ludique et animée, Health matters, parcourt les innovations médicales depuis 1950. Le musée de la Croix-Rouge,

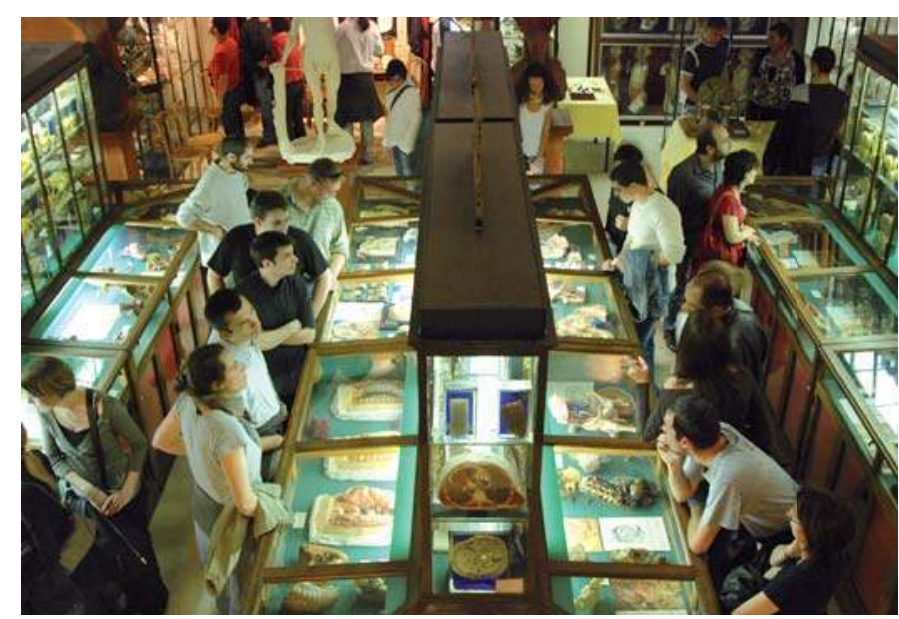

Le musée Testut Latariet à Lyon,

lors de la Nuit des musées, le 16 mai 2009 (c) Éric Le Roux/Service Communication UCBL/Musée d'Anatomie

dans le respect de sa mission de témoigner des conflits mondiaux, passés et actuels, traite des questions d'exclusion, d'enfermement et de brutalité. Sa scénographie reconstitue des lieux et des ambiances par l'intermédiaire des dispositifs audiovisuels et des installations multimédia. Le musée Notre-Dame-à-laRose, soutenu par les fonds européens, se dote d'une politique communautaire et sociale forte. L'Hygiene Museum focalise sur l'Homme et toutes ses expositions en découlent : le sommeil, les cinq sens, la guerre et la médecine, la sexualité, le bonheur, le cerveau, la mémoire, la vie et la mort, la nutrition.

\section{Conclusion : la justification d'un musée de la santé à Lyon?}

Malgré des exemples isolés et intéressants à retenir, ce tour d'horizon pointe l'évidence de l'absence, en France et même en Europe, d'un musée traitant de toutes les branches des sciences médicales ou des questions de santé dans une perspective sociale et économique actuelle et exploitant pleinement la muséographie contemporaine. Ce constat plaide pour la création d'un musée national de la Santé ainsi que son implantation à Lyon et conforte le commanditaire de l'étude de la pertinence de son exercice. La ville de Lyon dispose, en effet, de plusieurs atouts : un contexte économique, industriel, universitaire, hospitalier et scientifique d'envergure internationale ; des collections hospitalière, industrielle, universitaire exhaustives et de bonne facture ; un lieu d'accueil ; et un réseau de conservateurs et collectionneurs déjà à l'œuvre. 


\section{Notes}

(1) L'Hôtel-Dieu fait partie du périmètre inscrit au Patrimoine mondial de l'UNESCO en 1998.

(2) Professeur René Mornex, président du réseau de regroupement des musées de la santé à Lyon, président-suppléant du conseil d'administration des Hospices Civils de Lyon.

(3) Bruno Jacomy, directeur-adjoint du musée des Confluences à Lyon, a été mandaté à ce titre pour rédiger un rapport d'orientation Vers un musée de la santé à Lyon à la demande du professeur Mornex.

(4) En 2009, la connaissance du patrimoine de la santé se fonde principalement sur les études de Jacques Poisat, Gérard Tilles, Daniel Wallach et Bruno François.

(5) Jacques Poisat, président du Groupe de recherche interdisciplinaire sur les patrimoines de la santé

(6) Robert Bud, conservateur en chef des Sciences médicales au Science Museum, Londres

\section{Bibliographie}

Atkinson, W. Enquête préalable à la mise en place d'un musée de la santé à Lyon : un état des lieux. Lyon, 2009.

François, B. Présent et Avenir du Patrimoine Hospitalier Européen. 2001 http://europaphe.aphp.org/fr/home.html

Poisat, J. Les hôpitaux français et leur patrimoine. Charlieu : Éditions Musée de Charlieu, 1993.

Wallach, D. et Tilles, G. Les musées de médecines. Histoire, patrimoine, et grandes figures de la médecine en France. Toulouse : Privat 1999.

Pour une bibliographie plus complète, les lecteurs peuvent consulter la thèse professionnelle de l'auteure sur le site suivant :

www.ensam.eu/fr/centres_et_instituts/centre_cluny/bibliotheque/ressources 